\title{
DIVERGENCE INSTABILITY OF MULTIPLE-PARAMETER CIRCULATORY SYSTEMS*
}

\author{
BY \\ K. HUSEYIN AND H. H. E. LEIPHOLZ \\ University of Waterloo, \\ Waterloo, Ontario, Canada
}

\begin{abstract}
The free vibrations and stability of a discrete linear non-dissipative system subjected to the combined effect of several independent circulatory and conservative loads are studied. Attention is restricted to systems which exhibit only divergence-type instability and are incapable of flutter. Two theorems concerning the basic properties of the characteristic surfaces (loading-frequency relationship) and the stability boundary are established. One of the several other theorems insures that the stability boundary of the corresponding conservative system will always provide a lower bound for the actual stability boundary of the non-conservative system. This particular result is valid for all types of divergence-instability problems, including those which can exhibit flutter after divergence. The theorems established are quite general in the sense that they are valid for all systems in the class considered, without further analytical conditions. Practical implications of the general results are briefly discussed.
\end{abstract}

1. Introduction. The loading-frequency relationship is basic to an understanding of the instability behavior as well as of the dynamic response of a system at certain stages of loading. In a recent study [1] this relationship has been explored with regard to conservative multiple-eigenvalue problems, and two theorems concerning the convexity of the characteristic surfaces have been established. It has been observed that such general results have significant practical implications and can, in fact, be used to obtain upper and/or lower bounds for the critical loads and/or frequencies of the system.

The loading-frequency relationship gains even more significance when non-conservative forces are present, and it has been discussed by several authors $[2,3,4,5]$ with reference to a single loading parameter. The differing nature of instability behavior of conservative and various types of non-conservative systems can thus be explained conveniently. Flutter-type instability, for instance, occurs when a branch of the characteristic curve (loading-frequency) reaches a maximum at which two initially distinct roots of the characteristic equation coincide and, with a further increase of load, become a pair of complex conjugate roots. The divergence-type instability, on the other hand, occurs when the characteristic curves intersect the loading axis. Such an intersection can take place before a maximum is reached [5], resulting in divergence before flutter.

* Received June 1, 1971. 
There exists, however, another distinct class of non-conservative systems which are not capable of losing stability by flutter at all; the only form of instability they can exhibit is the divergence type. Pflüger's rod, which consists of a hinged bar under a uniformly distributed follower load, is an example. A column hinged at both ends and subjected to torsion is of this type $[3,6]$ also. Apparently the symmetric boundary conditions have an influence in shaping up the distinctive characteristics of this class of systems, but cannot be considered as decisive.

Leipholz [2] demonstrated analytically that the instability behavior of such systems is essentially the same as that of conservative systems, that the asymmetric matrix creating the non-conservativeness is symmetrizable, and that the roots of the characteristic equation are always real. Such systems can aptly be called 'pseudo-conservative'.

The aim of this paper is to explore the properties of the characteristic surfaces (the curves take the form of surfaces in the presence of more than one independent loading parameter) associated with this class of systems with particular reference to the stability boundary. The systems considered have several independent loading parameters which represent conservative and/or non-conservative circulatory loads. Damping and gyroscopic forces are assumed to be absent. The paper essentially extends the concepts introduced in [1] to circulatory systems and establishes the convexity of both the stability boundary and the first characteristic surface. Similar investigations have previously led to several theorems regarding the shape of the stability boundary of linear and non-linear conservative systems [7, 8, 9]. For the first time, this concept is extended to a class of non-conservative systems. In the analysis a conservative system corresponding to the original non-conservative system is defined, and it is proved that the stability boundary of the former provides a lower bound for the stability boundary of the latter. Various aspects of the interrelationship between the two systems are discussed, and several theorems are established. Finally, practical implications of the theory are discussed in some detail.

In a companion paper [10] some of the concepts of this work are discussed with respect to continuous one-dimensional systems.

2. System and basic concepts. We consider a linear, holonomic, autonomous dynamic system described by $N$ generalized coordinates $q_{i}(i=1,2, \cdots, N)$. The loading of the system is represented by $M$ independent parameters $P^{k}(k=1,2, \cdots, M)$. These parameters, in general, describe the circulatory forces, but some of them can represent conservative noncirculatory loads, to use Ziegler's terminology [3]. At this stage we need not draw a definite line between the two types of loads, but it must be kept in mind that the latter loads can be absent.

The generalized forces $Q_{i}=Q_{i}\left(P^{k}\right) ; i=1,2, \cdots, N, k=1,2, \cdots, M$, are assumed to be given as linear functions of the generalized coordinates $q_{i}$ :

$$
Q_{i}=P^{k} E_{i i}^{k} q_{i}
$$

where the summation convention is adopted both for subscripts and superscripts separately and will be employed in what follows. The constant matrices $E_{i j}^{k}$ are symmetric when the corresponding $P^{k}$ are conservative and asymmetric if nonconservative. For $P^{k}=0, k=1,2, \cdots$, let $q_{i}=d q_{i} / d t=0$ be the original equilibrium state of the system; then the kinetic energy is given as

$$
T=\frac{1}{2} m_{i} ; \dot{q}_{i} \dot{q}_{i}
$$


where $m_{i}$ is a constant, symmetric and positive definite matrix, and the dots indicate differentiation with respect to time, $t$.

The potential energy of the system is given by

$$
V=\frac{1}{2}\left(U_{i j} q_{i} q_{i}-P^{\alpha} E_{i j}^{\alpha} q_{i} q_{i}\right)
$$

where $\frac{1}{2} U_{i j} q_{i} q_{i}$ is the strain energy with a positive definite matrix $U_{i j}$, and $\alpha=1$, $2, \cdots M_{1}, M_{1}<M$. If the entire loading is circulatory, then (3) consists of the strain energy only. form

Lagrange's equations of motion yield $N$ homogeneous differential equations of the

$$
m_{i j} \ddot{q}_{i}+\left(U_{i i}-P^{k} E_{i j}^{k}\right) q_{i}=0
$$

which can also be written as

$$
m_{i j} \ddot{q}_{i}+A_{i j} q_{i}=0 .
$$

Here at least some of the $E_{i j}^{k}$ and consequently $A_{i j}$ are asymmetric. If the solutions are assumed to be in the form $q_{i}=a_{i} \exp (i \omega t)$, where $i=\sqrt{ }-1$, we obtain the characteristic equation as

$$
\operatorname{det}|D|=\operatorname{det}\left|-m_{i j} \Omega+U_{i j}-P^{k} E_{i j}^{k}\right|=0,
$$

where $\Omega=\omega^{2}$.

Our attention in the following sections (except for Sec. 5) will be focussed on systems whose characteristic equation (6) yields real (and normally distinct) roots, $\Omega$, under any combination of the loading parameters, $P^{k}$. If all the roots $\Omega_{i}>0$, the corresponding solutions are harmonic oscillations and thus bounded, implying stability. If one of them, say $\Omega_{k}$, vanishes, we have a critical state and when $\Omega_{k}<0$, the stability of the system is lost.

Eq. (6) describes certain $M$-dimensional hypersurfaces in the $(M+1)$-dimensional $\Omega-P^{k}$ space which was designated as the parameter space in [1], the $M$-dimensional loading space being a subspace of it. The surfaces (6) are called characteristic surfaces, the critical surfaces (defined in [7]) being their intersections with the hyperplane $\Omega=0$. The stability boundary [7] is associated with the first or fundamental characteristic surface (F.C.S.) consisting of points closest to the origin of the parameter space. The region containing the origin and bounded by F.C.S. is called the fundamental region.

Our attention will next be focussed on the basic properties of F.C.S.

3. Convexity of the fundamental characteristic surface. In the following analysis we shall operate on the matrix

$$
D=\left[-m_{i j} \Omega+A_{i i}\right]
$$

which is in general asymmetric, and certain properties of it must first be observed. It can readily be demonstrated that the eigenvalues of $D$ for any point $\left(\Omega_{0}, P_{0}^{k}\right)$ in the fundamental region are positive. To this end, suppose the positive definite $m_{i i}$ has been reduced to the unit matrix $I$ by two subsequent nonsingular congruence transformations, and (7) takes the form

$$
D=\left[-\delta_{i j} \Omega+\bar{A}_{i j}\right]
$$


where $\delta_{i j}$ is the Kronecker's delta. We can then reduce $\bar{A}$ to a triangular form $B_{i j}$ by an orthogonal transformation which yiclds

$$
D=\left[-\delta_{i j} \Omega+B_{i j}\right] .
$$

These matrix transformations are equivalent to a linear nonsingular transformation of the form $q_{i}=\alpha_{i j} u_{i}$. Clearly the determinant of (7), i.e., (6), vanishes whenever the determinant of (9) vanishes, and the characteristic roots of (6) are invariant under the nonsingular transformations performed. Thus, according to our initial assumption, (6), and consequently (9), have real distinct roots which now lie on the principal diagonal of the triangular matrix $B_{i i}$ and can be obtained from

$$
\left(B_{11}-\Omega\right)\left(B_{22}-\Omega\right) \cdots\left(B_{N N}-\Omega\right)=0 .
$$

Supposing that the roots are arranged in the ascending order

$$
B_{11}<B_{22}<\cdots<B_{N N},
$$

we can readily see, on the basis of (10), that for any loading $P^{k}=P_{0}^{k}(k=1,2, \cdots, M)$ and any $\Omega_{0}\left(P_{0}^{k}\right)<B_{11}\left(P_{0}^{k}\right)$ the eigenvalues of the matrix $D\left(\Omega_{0}, P_{0}^{k}\right)$ will be positive. We further note that $P_{0}$ and $\Omega_{0}\left(P_{0}^{k}\right)$ can be chosen anywhere in the fundamental region and can be negative (Fig. 1).
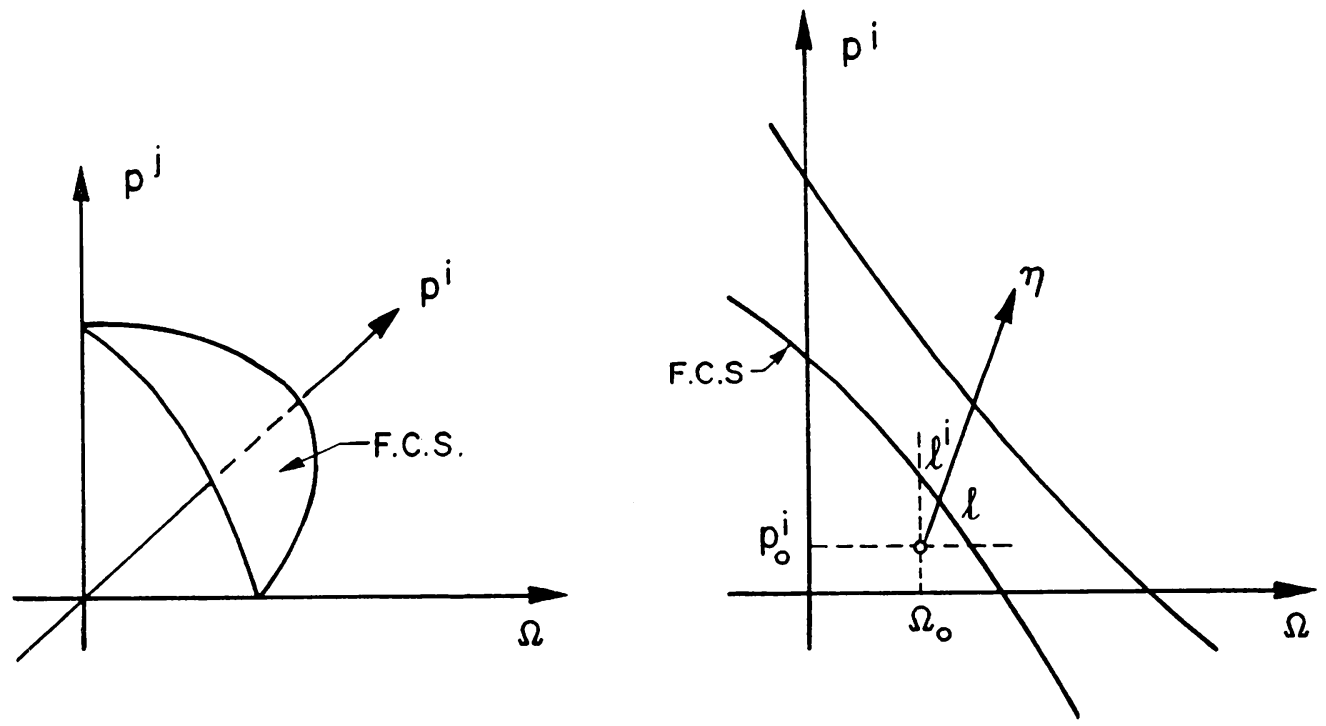

Fig. 1.

To demonstrate the convexity of F.C.S., consider an arbitrarily chosen point $\left(\Omega_{0}, P_{0}^{k}\right)$ in the fundamental region and a ray $\eta$ defined by

$$
P^{k}=P_{0}^{k}+l^{k} \eta, \quad \Omega=\Omega_{0}+l_{\eta},
$$

emerging from this point in an arbitrarily chosen direction determined by the direction cosines $\left(l^{k}, l\right)$. The radius vector $\eta$ is allowed to take only positive values. Introducing $(12 \mathrm{a}, \mathrm{b})$ into $(6)$, we get

$$
\operatorname{det}|D|=\operatorname{det}\left|C_{i j}\left(P_{0}^{k}, \Omega_{0}\right)-\eta F_{i j}\left(l^{k}, l\right)\right|
$$


where

$$
\begin{aligned}
& C_{i i}=U_{i j}-P_{0}^{k} E_{i j}^{k}-m_{i j} \Omega_{0}, \\
& F_{i j}=l^{k} E_{i j}^{k}+l m_{i j},
\end{aligned}
$$

and both $C_{i j}$ and $F_{i j}$ are asymmetric. We have, however, already observed that $C_{i j}$ has distinct positive eigenvalues, and hence it can be reduced to its diagonal form by a similarity transformation. Thus, supposing such a nonsingular matrix $T$ has been found, we have

$$
T^{-1} D T=T^{-1} C T-\eta T^{-1} F T
$$

where $T^{-1}$ is the inverse of $T$, and $T^{-1} C T$ is positive diagonal with positive elements $C_{i i}$.

Using the diagonal matrix $K=\operatorname{diag} .\left\{1 / \sqrt{ } C_{11}, 1 / \sqrt{ } C_{22}, \cdots, 1 / \sqrt{ } C_{N N}\right\}$ we further reduce $C_{i i}$ to the unit matrix $I$ :

$$
K T^{-1} D T K=I-\eta K T^{-1} F T K .
$$

A final orthogonal transformation with the orthogonal matrix $S$ reduces the asymmetric matrix $\left(K T^{-1} B T K\right)$ to a triangular form, leaving the unit matrix $I$ unchanged:

$$
S^{-1} K T^{-1} D T K S=I-\eta G,
$$

where $G$ is triangular with diagonal elements $G_{i i}$ which, under our basic assumption in this paper, are real and can be positive or negative. On the basis of the same assumption, we further note that, instead of insisting on an orthogonal transformation with $S$, a similarity transformation could also be performed such that $G$ takes a diagonal form rather than triangular.

The determinant (13) is now given as

$$
\text { b. } \operatorname{det}|D|=\operatorname{det}\left|I-\eta G_{i i}\right|
$$

where $b$ is a constant. Obviously $\operatorname{det}|D|$ vanishes whenever $\operatorname{det}\left|I-\eta G_{i i}\right|$ vanishes. In other words, the roots $\eta$ of (13) are invariant under the nonsingular transformations introduced.

Considering the variation of (19) with $\eta$, we see that for sufficiently small $\eta$, det $|D|>$ 0. Suppose at least one of the diagonal elements $G_{i i}$, say $G_{k k}$, is positive; as we increase the positive quantity $\eta$ from zero, the stage at which

$$
1-\eta G_{k k}=0
$$

will be reached, resulting in a zero determinant. At this point the ray intersects the F.C.S. If more than one $G_{i i}$ is positive, intersections with other characteristic surfaces are encountered as $\eta$ is further increased. All these uncoupled surfaces, however, will lie outside the fundamental region since $1-\eta G_{k k}<0$. If all $G_{i i}$ are negative, det $|D|$ remains positive as $\eta$ increases. Hence, no characteristic surface will be intersected.

Obviously, a ray in an arbitrary direction emerging from an arbitrarily chosen point in the fundamental region cannot intersect the F.C.S. more than once and if the fundamental region is once left, it cannot be re-entered by proceeding along the ray.

These arguments lead to the result that F.C.S. is a strictly convex surface whose concavity is toward the fundamental region (or origin) unless it takes the form of a flat plate. Thus, we have a theorem. 
Theorem 1. The fundamental characteristic surface cannot have convexity toward the fundamental region.

This result is similar to that obtained in [1] for conservative systems.

We now note that the stability boundary, being the intersection of F.C.S. with the hyperplane $\Omega=0$, will also obey the convexity law. This is a direct corollary of Theorem 1 , but due to its practical significance, it will be stated as a second theorem.

Theorem 2. The stability boundary cannot have convexity toward the region of stability.

4. The corresponding conservative system and lower bound theorems. The corresponding conservative system is defined as follows. Suppose all the asymmetric matrices $E_{i j}^{k}$ are expressed as the sum of their symmetric part $\stackrel{*}{E}_{i j}^{k}$ and skew-symmetric part $\bar{E}_{i j}^{k}$; the characteristic equation then has the form

$$
\operatorname{det}|D|=\operatorname{det}\left|-m_{i j} \Omega+U_{i i}-P^{k} E_{i i}^{*}-P^{k} \bar{E}_{i i}^{k}\right|=0 .
$$

The system obtained by ignoring the skew-symmetric parts $\bar{E}_{i j}^{k}$ will be called 'the corresponding conservative system' which has the characteristic equation

$$
\operatorname{det}|\stackrel{*}{D}|=\operatorname{det}\left|-m_{i j} \Omega+U_{i i}-P^{k} E_{i i}^{*}\right|=0 .
$$

The convexity of the F.C.S. of this system was proved in [1]. It was also observed that the matrix

$$
\stackrel{*}{D}=\left[-m_{i j} \Omega+U_{i j}-P^{k} \stackrel{*}{k}_{i j}^{k}\right]
$$

was positive definite for any point $\left(\Omega_{0}, P_{0}^{k}\right)$ in its own fundamental region. This region is shown shaded in Fig. 2.

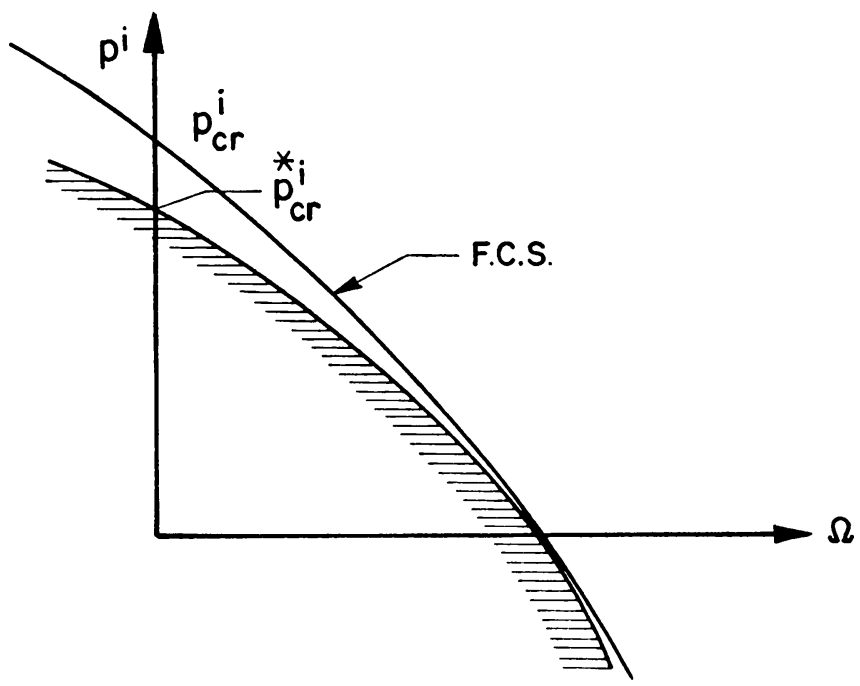

Fig. 2. 
Before proceeding further, we recall the mathematical fact that if $M_{i i}$ is a positive definite matrix, then,

$$
\operatorname{det}\left|M_{i i} \pm N_{i j}\right|>0,
$$

where $N_{i j}$ is any skew-symmetric matrix. This can readily be verified by associating the matrices with quadratic forms in certain arbitrary variables $x_{i}$ and observing that $N_{i} x_{i} x_{i}$ will always vanish (see also [11]). A more direct proof is given in the appendix.

Returning to our problem; we state the following lemma:

Lemma. The fundamental characteristic surface of the non-conservative system cannot enter into the fundamental region of the corresponding conservative system.

Proof: Suppose the F.C.S. of the non-conservative system has common points with the fundamental region of the corresponding conservative system, and one of these points is $\left(\Omega_{0}, P_{0}^{k}\right)$; then the matrix $\stackrel{*}{D}\left(\Omega_{0}, P_{0}^{k}\right)$ is positive definite and

$$
\operatorname{det}|\stackrel{*}{D}+\bar{D}| \equiv \operatorname{det}\left|-m_{i j} \Omega_{0}+U_{i j}-P_{0}^{k}{ }^{*} E_{i j}^{k}-P_{0}^{k} \bar{E}_{i j}^{k}\right|>0
$$

where $\bar{D}=-P^{k} \bar{E}_{i i}^{k}$ is skew-symmetric. Hence the point $\left(\Omega_{0}, P_{0}^{k}\right)$ cannot be on the F.C.S., since it should then satisfy $\operatorname{det}|\vec{D}+\bar{D}|=0$. This concludes the proof of the lemma.

We next note that for $P^{k}=0$ the two systems have a common point on the $\Omega$ axis which is the smallest natural frequency. Keeping this in mind, combining Theorem 1 with the similar convexity theorem for the corresponding conservative system [1], and in view of the lemma just proved we reach the following obvious conclusions:

TheOREm 3. The fundamental characteristic surface of the non-conservative system is tangent to the fundamental characteristic surface of the corresponding conservative system at the first natural frequency point on the $\Omega$ axis.

A valid question arises whether two surfaces have only one common point. This is definitely so if all parameters $P^{k}$ represent circulatory forces, but it will be seen in what follows that when some $P^{k}$ represent conservative forces, the two surfaces will be tangent along a curve or surface depending on the number of conservative loads.

Now, however, we reach another significant result on the basis of the theorems and lemma established above. It is clear (see Fig. 2) from the above results that the F.C.S. of the corresponding conservative system cannot intersect the $P^{i}$ axes above the intersection of the nonconservative system. Hence, we have another basic result:

Theorem 4. The stability boundary of the corresponding conservative system provides a lower bound for the stability boundary of the non-conservative system.

Similar arguments lead to

Theorem 5. If the F.C.S. of the corresponding conservative system takes the form of a flat plane, the F.C.S. of the non-conservative system is necessarily the same plane.

Corollary. If the F.C.S. of the corresponding conservative system is a flat plane, the stability boundaries of two systems coincide and also take the form of a plane.

The case in which some of the loads are conservative deserves further attention. 
Suppose only one of $P^{k}$, say $P^{1}$, is conservative: the intersection of the F.C.S. with the $P^{1}-\Omega$ plane is obviously a single curve for both systems (Fig. 3). The two F.C.S. will,

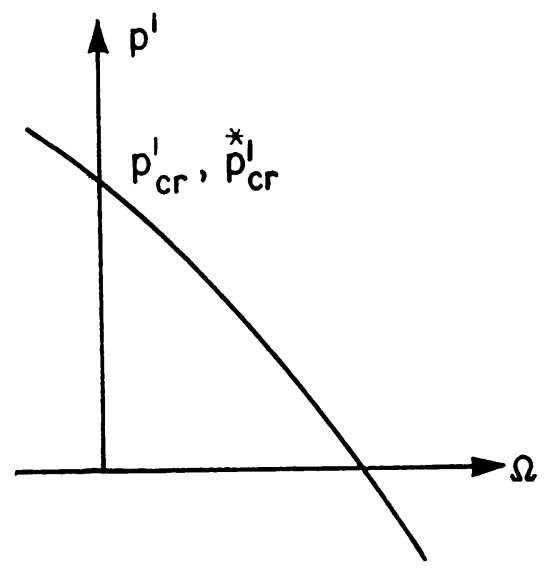

FIG. 3.

however, immediately separate as soon as the $P^{1}-\Omega$ plane is left. In other words, the two F.C.S. will be tangent along the characteristic curve associated with the conservative load, and this is true for any conservative load in the system. Thus, we have

Theorem 6. The two fundamental characteristic surfaces will have a common point, a line, or an $M$-dimensional surface according to whether the system has no, one or $M$ conservative load(s) respectively, and two surfaces are tangent at these common points.

A corresponding theorem can be stated for the stability boundaries of two systems. If the system has one or more conservative loads, the critical value of these loads are the same for both systems, but the critical values of circulatory loads will be different. thus, we can state the following theorem:

Theorem 7. The stability boundaries of two systems have nothing, a point, a curve or an $(M-1)$-dimensional surface in common according to whether the system has no, one, two or $M$ conservative load(s), respectively.

Fig. 4a illustrates schematically the above phenomena for $P^{1}$ and $P^{2}$ both conservative, Fig. $4 \mathrm{~b}$ for $P^{\prime}$ conservative and $P^{2}$ circulatory, and Fig. $4 \mathrm{c}$ for $P^{1}$ and $P^{2}$ both circulatory.

5. Divergence in flutter-type problems. The systems whose characteristic equations yield complex roots and the characteristic curves have maximums are essentially associated with flutter-type instabilities. Under the circumstances illustrated in Fig. 5, however, divergence instability may occur before the flutter load is reached. Even this type of divergence problem obeys the lower bound Theorem 4 . To observe this we only need to point out that in proving the Lemma of Sect. 4, we have made no assumptions with regard to the system considered to exclude this type of divergence. It is, therefore, evident that the characteristic surface of the non-conservative system (Fig. 5) cannot 


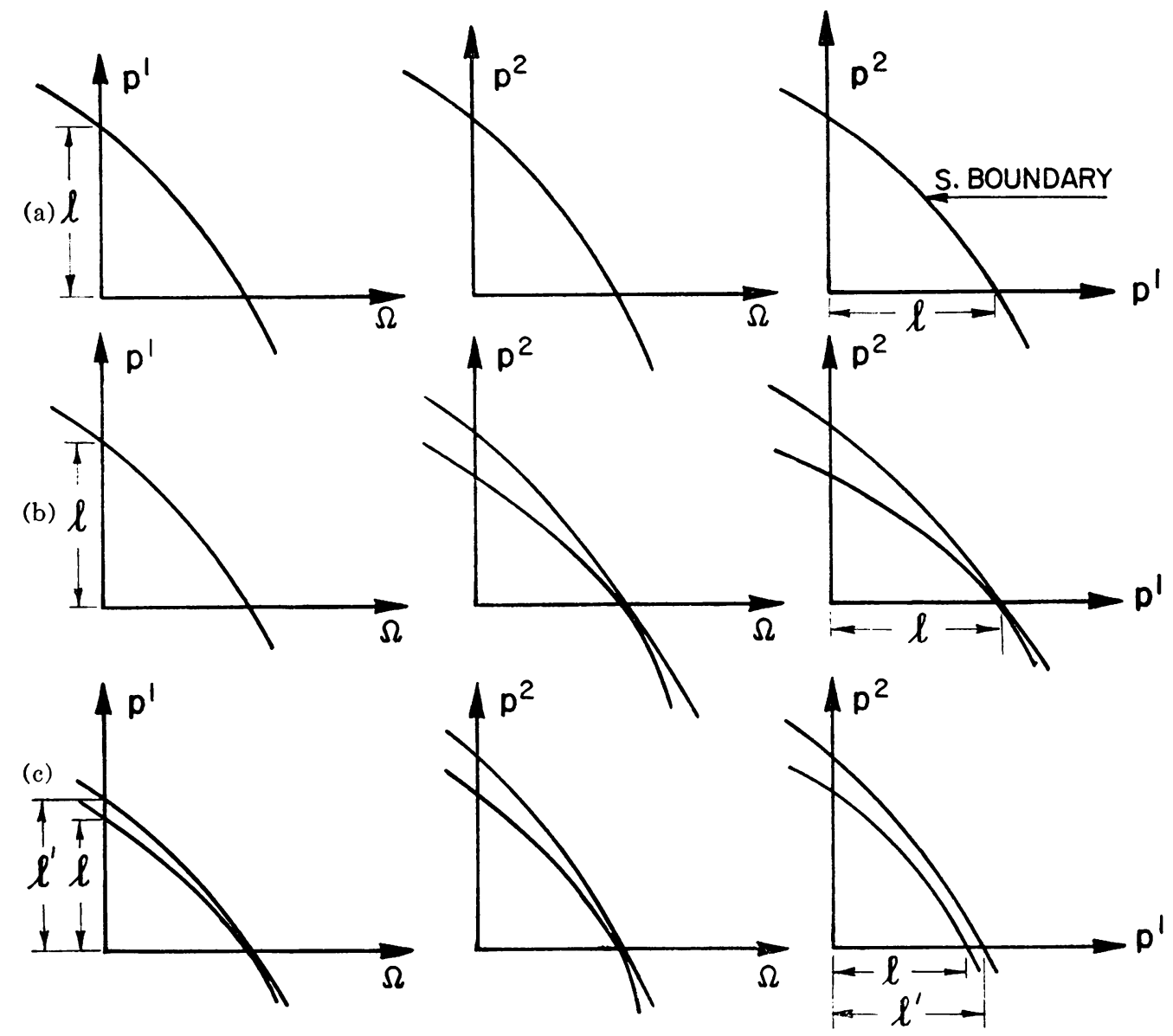

Fig. 4.

intersect the loading axes below the interaction points of the corresponding conservative system. Thus, we can make a stronger statement than Theorem 4:

Theorem 8. In all divergence-type instability problems the stability boundary of the corresponding conservative system provides a lower bound for the stability boundary of the non-conservative system.

6. Practical considerations. Theorem 1, establishing that the fundamental characteristic surface is concave towards the origin (if it is not a flat plane), can be used in two ways: either to estimate the frequencies at certain values of loading parameters or, alternatively, to estimate the stability boundary of the system.

In the former case, suppose any two points on the surface, say the first natural frequency and a critical load, are known. The straight line segment joining these two points is then a lower bound of the frequencies at any stage of loading. If the whole stability boundary is known, then the surface generated by the straight line passing through the known point on the $\Omega$ axis (natural frequency) and tracing the stability 


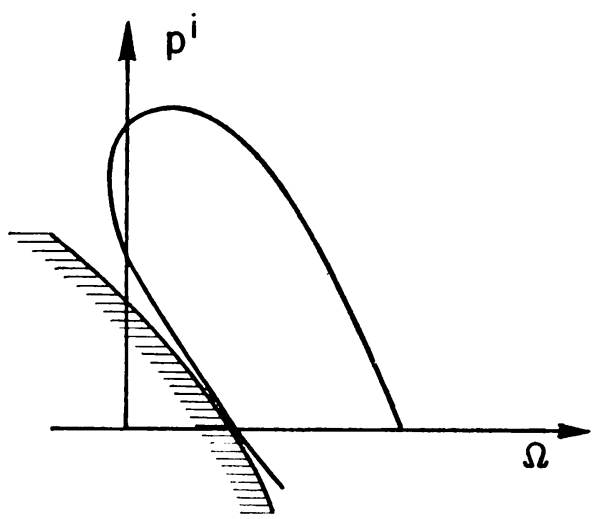

Fig. 5.

boundary constitutes a lower bound for the frequencies. Other possibilities exist and are discussed in [1].

The alternative application of Theorem 1 is to obtain an upper bound for various critical loads. Thus, if any two points on the F.C.S. are known, the intersection of the straight line joining them with the loading space yields upper bounds for various critical combinations of loads. We shall refer the reader again to [1] for further discussion on this point.

Theorem 2 is a first extension of similar results existing for conservative systems to the non-conservative field. It can prove very useful in obtaining lower bounds for the stability boundary of a non-conservative system under the combined effect of independent conservative and circulatory loads. In fact, one can obtain lower bounds of the stability boundary without any analytical effort by merely using the results already existing in the literature.

Let us consider a hinged bar under the combined effect of an axial conservative load $P^{\prime}$ and a uniformly distributed follower load $P^{2}$ (Fig. 6). In the absence of $P^{2}$, the Euler load $P_{\mathrm{cr}}^{\prime}=\pi^{2} E I / l^{2}$, and in the absence of $P^{\prime}$ the critical load $P_{\mathrm{cr}}^{2}=18.96\left(E I / l^{3}\right)$ [12] is known. Under the combined effect of $P^{1}$ and $P^{2}$, the critical interaction curve (stability boundary) is concave towards the origin (Theorem 2), and by joining the

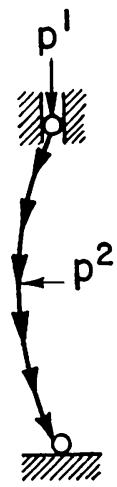

Fig. 6. 
two points $P_{\mathrm{or}}^{1}$ and $P_{\mathrm{or}}^{2}$ we immediately get a lower bound of it. Several other examples can be quoted.

We now proceed, however, to discuss the practical implications of the remaining results with particular reference to Theorems 4 and 8. Suppose we have a non-conservative problem in hand, and we know that the system can lose its stability by flutter. We can then obtain a lower bound for the stability boundary (or for the critical load if there is just one circulatory load) by using the results of the corresponding conservative system. Such results may already exist in the literature or can be obtained through much simpler analytical work. The Pflüger's rod is again a good example, demonstrating how close the lower bound can be. In this example, the corresponding conservative system with uniformly distributed constant-directional force (instead of tangential force) has a critical load $P_{\text {cr }}=18.46\left(E I / l^{3}\right)$, compared to $P_{\text {cr }}=18.96\left(E I / l^{3}\right)$ of the nonconservative system. This example alone gives an idea about the significance of Theorems 4 and 8. Other examples are also available [12]. We must remark here that the loading of the corresponding conservative system is not necessarily the conservative component of the circulatory load, but this happens to be the case in many problems of the class as a result of the symmetric boundary conditions.

The lower bound will be the exact solution if Theorem 5 is satisfied. Such a situation takes place when the equations of the corresponding conservative system uncouples naturally without introducing transformations, thus resulting in a F.C.S. in the form of a flat plane. The two systems, then, become essentially identical.

From a dynamics point of view Theorems 3,4 and 5 can also be helpful in estimating the frequencies of the non-conservative system on the basis of the corresponding conservative system.

Similar considerations are valid for Theorems 6 and 7, and their interpretation will be left to the reader. It is hoped that the general results of this paper will be useful in estimating critical loads and/or frequencies of non-conservative systems in the class considered.

We now give some guidelines for the identification of this class of systems.

Mathematical description of the system. The class of non-conservative systems considered in this paper (except for Sec. 5) behave in the same manner as conservative systems. Mathematically, they are associated with a characteristic equation which yields real roots under all loading situations. The asymmetric matrix involved is sym metrizable, yielding real roots just like a symmetric matrix. The real roots are normally distinct in structural field.

Generally speaking, suppose $\bar{A}$ is an asymmetric but symmetrizable matrix; the following are, then, equivalent conditions characterizing such matrices [13, 14]:

1. $\bar{A}=H D$, where $H$ and $D$ are symmetric and one of them, say $D$, is positive definite.

2. $\bar{A}=U^{-1} S U$, where $S$ and $U$ are symmetric and $U$ is positive definite. This result can readily be derived from the first condition. Thus, $\bar{A}=H D^{1 / 2} D^{1 / 2}$, or $D^{1 / 2} \bar{A} D^{-1 / 2}=$ $D^{1 / 2} H D^{1 / 2}$, or $U \bar{A} U^{-1}=S$, where $U=D^{1 / 2}$ and $S=D^{1 / 2} H D^{1 / 2}$, a symmetric matrix [13].

3. $\bar{A}^{\prime}=T^{-1} \bar{A} T$, where $T$ is symmetric and positive definite and $\bar{A}^{\prime}$ is the transpose of $\bar{A}$. This condition follows the second one and can also readily be verified.

Condition 2 shows that $\bar{A}$ is "similar" to a symmetric matrix $S$. In the theory of matrices it is well known that the similar matrices have the same characteristic roots. Hence, $\bar{A}$ and $S$ have the same roots which are necessarily real. 
Two-degree-of-freedom system. In many practical problems the differential equations of motion will result in a characteristic equation which is effectively reduced to that of a two-degree-of-freedom system. It will, therefore, be very helpful to identify the system in simplest possible way.

Suppose $\bar{A}$ is an asymmetric matrix; its characteristic equation

$$
\operatorname{det}\left|\begin{array}{cc}
\bar{A}_{11}-\lambda & \bar{A}_{12} \\
\bar{A}_{21} & \bar{A}_{22}-\lambda
\end{array}\right|=0
$$

has the discriminant

$$
\Delta=\left[\left(A_{11}+A_{22}\right)^{2}-4 A_{11} A_{22}+4 A_{12} A_{21}\right]^{1 / 2}
$$

or

$$
\Delta=\left[\left(A_{11}-A_{22}\right)^{2}+4 A_{12} A_{21}\right]^{1 / 2} .
$$

Evidently a sufficient condition that the roots be real is given by $\operatorname{sign} A_{12}=\operatorname{sign} A_{21}$. Such a simple condition can readily be verified. Boundary conditions can also give indications about the class of the system in hand. Once the system is identified as one of the class considered, then, the theorems are expected to reduce the analytical work to a minimum.

Appendix. We shall prove that

$$
\operatorname{det}\left|M_{i j} \pm N_{i j}\right|>0,
$$

where $M_{i j}$ is symmetric positive definite and $N_{i j}$ is skew-symmetric.

An orthogonal matrix $P$ can be found to reduce $M$ to its diagonal form

$$
\bar{M} \pm P^{\prime} N P,
$$

where $\bar{M}$ is diagonal and $P^{\prime} N P$ is skew-symmetric. Using the matrix

$$
K=\operatorname{diag}\left\{1 / \sqrt{ } \bar{M}_{11}, 1 / \sqrt{ } \bar{M}_{22}, \cdots\right\}
$$

we get

$$
K \bar{M} K \pm K P^{\prime} N P K=I \pm K P^{\prime} N P K,
$$

where $I$ is the unit matrix and $K P^{\prime} N P K$ is still skew-symmetric. An orthogonal matrix $U$ can then be found to canonize this last skew-symmetric matrix,

$$
\begin{aligned}
I+ & U^{\prime} K^{\prime} P^{\prime} N P K U=I \pm \operatorname{diag}\left\{\left[\begin{array}{cc}
0 & \mu_{1} \\
-\mu_{1} & 0
\end{array}\right],\left[\begin{array}{cc}
0 & \mu_{2} \\
-\mu_{2} & 0
\end{array}\right], \cdots\right\} \\
& \equiv I \pm D
\end{aligned}
$$

obviously,

$$
\operatorname{det}|M \pm N|=|I \pm D| \cdot|K|^{-2}|P|^{-2} \cdot|U|^{-2}>0 .
$$

REFERENCES

[1] K. Huseyin and J. Roorda, The loading-frequency relationship in multiple eigenvalue problems, J. Appl. Mech. 38-4, series E, 1007-1011 (1971)

[2] H. H. E. Leipholz, Stability theory, Academic Press, Inc., New York and London, 1970 
[3] H. Ziegler, Principles of structurai stability, Blaisdell Publishing Co., Waltham, Mass., 1968

[4] V. V. Bolotin, Nonconservative problems of the theory of elastic stability, Moscow, 1961

[5] G. Herrmann and R. W. Bungay, On the stability of elastic systems subjected to nonconservative forces, J. Appl. Mech. 31, 435-440 (1964)

[6] H. H. E. Leipholz, Uber ein Kriterium für die Gülligheit der Statischen Methode zur Bestimmung der Knicklast von elastischen Stäben unter nichtkonservativer Belastung, Ing. Arc. 32, S. 286-296 (1963)

[7] K. Huseyin, The elastic stability of structural systems with independent loading parameters, Int. J. Solids Struct. 6, 677-691 (1970)

[8] K. Huseyin, The convexity of the stability boundary of symmetric systems, Acta Mech. 8, 205-221 (1969)

[9] K. Huseyin, The stability boundary of systems with one degree of freedom, part I, Meccanica 5, 306-311 (1970)

[10] H. H. E. Leipholz and K. Huseyin, On the slability of one-dimensional continuous systems with polygenic forces, Meccanica VI, 4, 253-257 (1971)

[11] S. N. Prasad and G. Herrmann, Some theorems on stability of discrete circulatory systems, Acta Mech. 6, 208-216 (1968)

[12] H. H. E. Leipholz, Application of Liapunov's direct method to the stability problem of rods subjected to follower forces, SMD Report No. 26, December 1969

[13] O. Taussky, Positive definite matrices and their role in the study of characteristic roots of general matrices, Advances in Mathematics 2, 175-186, Academic Press, 1968

[14] R. Zurmühl, Matrices, Springer, Berlin, 1958

[15] K. Huseyin, Instability of symmetric structural systems with independent loading parameters, Quart. Appl. Math. 28, 571-586 (1970) 